\title{
Prostate-specific membrane antigen and renal cell carcinoma: a new diagnostic and therapeutic target?
}

\author{
Federica Matteucci, Giovanni Paganelli \\ Nuclear Medicine Unit, Istituto Romagnolo per lo Studio e la Cura dei Tumori (IRST), Meldola (Fc), Italy \\ Correspondence to: Giovanni Paganelli. Nuclear Medicine Unit, Istituto Romagnolo per lo Studio e la Cura dei Tumori (IRST), Meldola (Fc), Italy. \\ Email: giovanni.paganelli@irst.emr.it. \\ Comment on: Spatz S, Tolkach Y, Jung K, et al. Comprehensive Evaluation of Prostate Specific Membrane Antigen Expression in the Vasculature of \\ Renal Tumors: Implications for Imaging Studies and Prognostic Role. J Urol 2018;199:370-7.
}

Submitted Nov 23, 2017. Accepted for publication Nov 28, 2017.

doi: $10.21037 /$ tcr.2017.12.02

View this article at: http://dx.doi.org/10.21037/tcr.2017.12.02

The prostate-specific membrane antigen (PSMA) is a membrane glycoprotein (type II-carboxypeptidase) encoded by the FOLH1 gene, which is hyper-expressed in prostate cancer (PCa). 68Ga-PSMA PET/CT is a new diagnostic tool for the localization of $\mathrm{PCa}$ foci in patients with biochemical recurrence. Its use in presurgical staging as well as in early treatment evaluation is also under investigation (1). Moreover, the possibility of labeling PSMA antagonists with radionuclides emitting $\alpha$ and/or $\beta$-particles is becoming an interesting therapeutic application for the same molecule (2). However, PSMA, despite its name, is not really specific for $\mathrm{PCa}$ as it is also expressed by endothelial cells in the neovascular tissue of many solid tumors including kidney cancer. It has also been reported that the expression of PSMA in normal renal parenchyma can be detected within the brush borders and apical cytoplasm in a subset of proximal tubules $(3,4)$.

Recently, Spatz et al. (5) evaluated PSMA immunohistochemical expression in neovascularized tissue in a cohort of 257 patients with renal cell carcinoma (RCC), mostly clear cell RCC (ccRCC) and, to a lesser extent, papillary RCC (pRCC) and chromophobe RCC (chRCC). The authors correlated PSMA expression with clinicalpathological parameters related to 68Ga-PSMA PET/CT. They also investigated the possible prognostic role of FOLH1 (folate hydrolysis 1) gene encoding for PSMA in patients with ccRCC and pRCC. Results of immunohistochemical analysis revealed that PSMA hyperexpression was only present in the endothelium of neovascular tissue in RCC samples. In particular, $82.5 \%$ of ccCRCC and $71.4 \%$ of chRCC samples expressed PSMA glycoprotein, whereas only $13.6 \%$ of pRCC showed PSMA staining.

For the first time, Spatz et al. highlighted a significant correlation between increasing levels of PSMA expression and overall survival among patients with ccRCC. The association between PSMA expression and overall survival also maintained its significance (HR 2.02; 95\% CI: 1.08-3.79) after correlation with key clinical features such as tumor grade, primary tumor stage and metastases. These results were largely supported by the data analysis of RNA expression from the Cancer Genome Atlas (TCGA). The authors reported a significant correlation between the expression of FOLH1 mRNA and survival in both univariate and multivariate analysis in pRCC patients, whereas it was only significant in univariate analysis in ccRCC patients. These results allow us to hypothesize an important role of PSMA in the management of RCC patients.

Recently, a potential role of $68 \mathrm{Ga}$-PSMA PET/CT was reported in the preoperative evaluation of patients with RCC $(6,7)$. The main advantage of 68Ga-PSMA PET/CT compared to conventional methods, in particular CT, lies in its ability to detect small lymph node lesions that do not exceed CT volumetric limit. In addition, the possibility of performing a whole body scan enables distant metastases to be detected.

PSMA could also be used to evaluate response to therapy in RCC. At present, inhibition of angiogenesis represents a new treatment strategy for RCC.

It is estimated that more than 300 compounds are currently being investigated for their potential antiangiogenic effect, and a large series of inhibitors of angiogenesis have shown great potential in (pre)clinical studies for the treatment of many tumors.

Sorafenib and sunitinib, two tyrosine kinase inhibitors of the VEGF and PDGF receptor, were recently registered for the treatment of metastatic RCC.

68Ga-PSMA PET/CT could be a triage test for 
antiangiogenic therapy and for subsequent evaluation of the response, monitoring the variations of "PSMA expression" as a surrogate of neo-angiogenesis in vivo. To date, only one study performed in eight RCC patients revealed a potential role of $68 \mathrm{Ga}$-PSMA PET/CT in the evaluation of early response to systemic therapy compared to MRI and CT (8).

According to Spatz et al., PSMA may represent a molecule to be used as both a diagnostic and therapeutic agent in RCC. The latter application could be very important if we consider that RCC remains largely incurable, despite the increasing number of currently available drugs.

Like PCa, the possibility of labeling PSMA antagonists with $\alpha$ and/or $\beta$ particles $(9,10)$ could also pave the way for an effective radionuclide therapy in patients with RCC.

Although there is still a long way to go, in our opinion we are heading in the right direction. However, large-scale prospective studies are warranted.

\section{Acknowledgments}

Funding: None.

\section{Footnote}

Provenance and Peer Review: This article was commissioned and reviewed by the Section Editor Peng Zhang (Department of Urology, Zhongnan Hospital of Wuhan University, Wuhan, China).

Conflicts of Interest: Both authors have completed the ICMJE uniform disclosure form (available at http://dx.doi. org/10.21037/tcr.2017.12.02). The authors have no conflicts of interest to declare.

Ethical Statement: The authors are accountable for all aspects of the work in ensuring that questions related to the accuracy or integrity of any part of the work are appropriately investigated and resolved.

Open Access Statement: This is an Open Access article distributed in accordance with the Creative Commons Attribution-NonCommercial-NoDerivs 4.0 International License (CC BY-NC-ND 4.0), which permits the noncommercial replication and distribution of the article with the strict proviso that no changes or edits are made and the original work is properly cited (including links to both the formal publication through the relevant DOI and the license). See: https://creativecommons.org/licenses/by-nc-nd/4.0/.

\section{References}

1. von Eyben FE, Picchio M, von Eyben R, et al. (68)GaLabeled Prostate-specific Membrane Antigen Ligand Positron Emission Tomography/Computed Tomography for Prostate Cancer: A Systematic Review and Metaanalysis. Eur Urol Focus 2016. [Epub ahead of print].

2. Rahbar K, Ahmadzadehfar H, Kratochwil C, et al. German Multicenter Study Investigating 177Lu-PSMA-617 Radioligand Therapy in Advanced Prostate Cancer Patients. J Nucl Med 2017;58:85-90.

3. Baccala A, Sercia L, Li J, et al. Expression of prostate-specific membrane antigen in tumor-associated neovasculature of renal neoplasms. Urology 2007;70:385-90.

4. Lopes AD, Davis WL, Rosenstraus MJ, et al. Immunohistochemical and pharmacokinetic characterization of the site-specific immunoconjugate CYT-356 derived from antiprostate monoclonal antibody 7E11-C5. Cancer Res 1990;50:6423-9.

5. Spatz S, Tolkach Y, Jung K, et al. Comprehensive Evaluation of Prostate Specific Membrane Antigen Expression in the Vasculature of Renal Tumors: Implications for Imaging Studies and Prognostic Role. J Urol 2018;199:370-7.

6. Sawicki LM, Buchbender C, Boos J, et al. Diagnostic potential of PET/CT using a (68)Ga-labelled prostatespecific membrane antigen ligand in whole-body staging of renal cell carcinoma: initial experience. Eur J Nucl Med Mol Imaging 2017;44:102-7.

7. Rhee H, Blazak J, Tham CM, et al. Pilot study: use of gallium-68 PSMA PET for detection of metastatic lesions in patients with renal tumour. EJNMMI Res 2016;6:76.

8. Siva S, Callahan J, Pryor D, et al. Utility of (68) Ga prostate specific membrane antigen - positron emission tomography in diagnosis and response assessment of recurrent renal cell carcinoma. J Med Imaging Radiat Oncol 2017;61:372-8.

9. Kratochwil C, Bruchertseifer F, Giesel FL, et al. 225AcPSMA-617 for PSMA-Targeted $\alpha$-Radiation Therapy of Metastatic Castration-Resistant Prostate Cancer. J Nucl Med 2016;57:1941-4.

10. Fendler WP, Rahbar K, Herrmann K, et al. (177)LuPSMA Radioligand Therapy for Prostate Cancer. J Nucl Med 2017;58:1196-200.

Cite this article as: Matteucci F, Paganelli G. Prostate-specific membrane antigen and renal cell carcinoma: a new diagnostic and therapeutic target? Transl Cancer Res 2018;7(Suppl 1):S24S25. doi: $10.21037 /$ tcr.2017.12.02 